\title{
FRECUENCIA DE PÉRDIDA DE LA COLA EN UN ENSAMBLE DE LAGARTIJAS DE OAXACA, MÉXICO
}

\author{
AARón GARCÍA-ROSALES ${ }^{1, *}$ y MATÍAs MARTÍNEZ-CORONEL ${ }^{2}$ \\ ${ }^{1}$ Universidad Autónoma Metropolitana-Iztapalapa. Av. San Rafael Atlixco 186, Col. Vicentina, Iztapalapa. 09340. \\ <aarongarciar23@gmail.com> \\ ${ }^{2}$ Universidad Autónoma Metropolitana-Iztapalapa. Av. San Rafael Atlixco 186, Col. Vicentina, Iztapalapa. 09340. \\ <marti17@hotmail.com> \\ *Autor de correspondencia
}

Recibido: 29/02/2016; aceptado: 22/04/2016

García-Rosales, A. \& Martínez-Coronel, M. 2016. Frecuencia de pérdida de la cola en un ensamble de lagartijas de Oaxaca, México. Acta Zoológica Mexicana (n. s.), 32(2): 174-181.

RESUMEN. El presente estudio evaluó la influencia de la edad, el sexo y el hábitat sobre la frecuencia de pérdida de la cola en tres especies de lagartijas endémicas a México. Los taxa tratados son Sceloporus bicanthalis, $S$. subpictus y Mesaspis gadovii, las cuales son simpátricas en las montañas de los Valles Centrales de Oaxaca. Las diferencias en la frecuencia de pérdida de la cola fueron evaluadas por sexo, edad, hábitat y especie mediante una prueba de Chi cuadrada en un tamaño de muestra de 259 organismos recolectados entre abril de 2010 y marzo de 2011. Los resultados indican que la estructura del hábitat, el sexo o la edad de los individuos (excepto en M. gadovii) no influyeron de manera significativa en la frecuencia de mutilación de la cola en las tres especies analizadas. Sin embargo, se presentaron diferencias significativas entre especies, estas diferencias pueden ser consecuencia del comportamiento agonístico propio de cada especie, y de una presión de depredación diferente para cada taxon.

Palabras clave: Autotomía caudal, especies endémicas, comportamiento agonístico, Oaxaca.

\section{INTRODUCCIÓN}

Entre los vertebrados, los reptiles tienen la capacidad de regenerar parte de sus estructuras corporales, estas capacidades son consideradas intermedias por ser menores a las de los ciclóstomos, peces y anfibios, pero superiores a las de aves y mamíferos (Alibardi 2010). La capacidad regenerativa no es homogénea en todos los grupos, ya que en lagartijas y tuataras está mejor representada, mientras que en serpientes, tortugas y cocodrilos es limitada, y es ausente en anfisbénidos (Alibardi 2010; Bateman \& Fleming 2009; Clause \& Capaldi 2006). Las lagartijas representan el grupo con mayor capacidad regenerativa, ya que éstos pueden restaurar sus tejidos, incluyendo el nervioso, partes del arco inferior mandibular y maxilar, de hecho algunas especies incluso pueden regenerar una extremi-
García-Rosales, A. \& Martínez-Coronel, M. 2016. Frequency of tail loss in assembly lizards of Oaxaca, Mexico. Acta Zoológica Mexicana (n. s.), 32(2): 174-181.

ABSTRACT. The present study evaluated the influence of age, sex and habitat on the frequency of tail loss in three species of endemic lizards to Mexico. The treated taxa are Sceloporus bicanthalis, S. subpictus and mesaspis gadovii and are sympatric in the mountains of the Central Valley of Oaxaca. The differences in the frequency of tail loss were evaluated by sex, age, habitat and species by Chi square test on a sample size of 259 organisms collected between April 2010 and March 2011. The results indicate that habitat structure, sex or age of individuals (except in Mgadovii) did not influence significantly in the frequency of mutilation of the tail in the three species analyzed. However, significant differences between species occur, these differences may be the result of agonistic behavior of each species, and of a pressure of predation different for each taxon.

Key words: Tail autotomy, endemic species, agonistic behavior, Oaxaca.

dad, pero son más conocidos porque la mayoría de ellos pierden la cola por autotomía (fenómeno por el cual algún individuo se deprende de alguna parte de su cuerpo provocada por él mismo a partir de ciertas zonas de ruptura, donde posteriormente se iniciará un proceso de regeneración) y son capaces de regenerarla (Alibardi 2010; Bateman \& Fleming 2009; Clause \& Capaldi 2006). La cola regenerada es similar en su aspecto externo a la original, pero internamente es más simple, ya que las vértebras se sustituyen por un tubo cartilaginoso calcificado, y la médula espinal, al igual que los músculos son rudimentarios (Alibardi 2010).

Bateman \& Fleming (2009) registraron la autotomía en 14 de 18 familias de lagartos, y encontraron que es un fenómeno raro en la mayoría de los taxa de talla grande, mientras que, en familias con miembros de talla mediana 
a pequeña, la presencia de autotomía es variable, ya que dentro de una misma familia existen taxa que poseen y otras que carecen de ella, como es el caso en especies de las familias Anguidae, Phrynosomatidae, Polychrotidae y Scincidae. La autotomía y regeneración de la cola en los lagartos, parece haber sido un proceso seleccionado a favor de la supervivencia, debido a las funciones variadas que este órgano cumple, ya que sirve como zona de almacenamiento de sustancias de reserva, puede funcionar como otra extremidad, como arma de defensa, órgano de equilibrio, punto de apoyo, distractor o señuelo (Clause \& Capaldi 2006; Pianka \& Vitt 2003). En el caso de los lagartos que son incapaces de desprenderse de la cola, Bateman \& Fleming (2009) plantean que este hecho puede estar relacionado a limitaciones adaptativas y a la historia filogenética de cada clado, pero sobretodo con el papel que la cola desempeña en cada especie. Por ejemplo, todas las especies de camaleones (Chamaleonidae) carecen de autotomía, hecho que puede estar asociado con la función prensil de la cola, que en este grupo es vital por sus hábitos trepadores (Vitt \& Caldwell 2009).

La autotomía caudal representa una ventaja para los organismos que la poseen, ya que este fenómeno salva momentáneamente la vida de un individuo, pero también implica un riesgo para la vida futura, ya que representa un costo ecológico y energético que se debe afrontar y resolver mientras se recupera el tejido perdido. Mientras la nueva cola se regenera, los organismos carecen temporalmente de las ventajas que otorga su presencia. Entre las consecuencias que se han identificado en los lagartos por perder la cola, se pueden citar la disminución de la tasa de crecimiento, la fecundidad y el status social (Dial \& Fitzpatrick 1981; Fox et al. 1998). Asimismo, los animales mutilados tienen menor probabilidad de conseguir pareja, menor éxito de defensa y evasión a los depredadores, su rendimiento locomotor y los niveles de actividad se modifican, el forrajeo, el uso del microhábitat y el comportamiento en general es afectado de manera negativa (Bateman \& Fleming 2009; Clause \& Capaldi 2006; Wilson 1992). Sin embargo, no en todos los taxa el afecto por perder la cola es el mismo, pues las consecuencias dependerán de la función que este órgano cumple en cada uno de ellos. Por ejemplo, en Cnemidophorus ( = Aspidoscelis) sexlinetus, Cophosaurus texanus, Podarcis muralis, Scincella lateralis y Uma notata se encontró que perder la cola entorpece la locomoción y reduce la velocidad de escape (Ballinger et al. 1979; Brown et al.1995; Dial 1984; Punzo 1982); mientras que en Uta stansburiana disminuyó su rango social (Fox et al. 1998; Fox \& Rostker 1982); en Coleonyx brevis, Lacerta monticola, Mabuya heathi, Psammodromus algiricus, y U. stansburiana, se observó una reducción en el éxito reproductor (Dial \& Fitzpatrick 1981; Fox \& McCoy 2000; Martin \& Salvador 1993; Salvador et al. 1996; Vitt 1981); en Psammodromus algiricus la captura de presas fue afectada negativamente (Martin \& Avery 1997), mientras que en S. lateralis, el comportamiento defensivo se modificó (Formanowicz et al. 1990a).

El estudio sobre la mutilación de la cola en lagartos es un tema interesante por los efectos que conlleva este fenómeno. Sin embargo, la mayoría de los estudios se han realizado con especies de Norteamérica, Europa y de Sudamérica; mientras que los trabajos con especies mexicanas han sido escasos (Castañeda et al. 2011; García-de la Peña et al. 2004; Leyte-Manríquez et al. 2007; OrtegaRubio et al. 2000; Ortega-Rubio \& Arriaga 1990). Por lo que, el presente estudio aporta información acerca de la influencia de la edad, el sexo y la cobertura vegetal sobre la frecuencia de mutilación de la cola en tres especies endémicas de lagartijas de México. Los modelos de especies son Sceloporus bicanthalis (Smith, 1937) y $S$. subpictus (Lynch \& Smith 1965) miembros de la familia Phrynosomatidae (Wiens et al. 2010) y Mesaspis gadovii (Boulenger 1913) que pertenece a la familia Anguidae (Good 1998). Las tres especies son diurnas, heliotérmicas y usan el microhábitat del suelo del bosque y pastizal. Sceloporus bicanthalis se distribuye en la tierras altas de la Sierra Madre Oriental, en los estados de Veracruz, Hidalgo, Tlaxcala, Estado de México, Puebla y centro de Oaxaca (Leaché et al. 2013; Wiens et al. 2010); S. subpictus se restringe a las montañas del centro de Oaxaca (Köhler \& Heimes 2002) y M. gadovii se encuentra en la Sierra Madre del Sur, en los estados de Guerrero y Oaxaca (Good 1988). Las tres especies son simpátricas en el área de estudio, ubicada en las montañas de los Valles Centrales de Oaxaca.

\section{MÉTODO}

Área de estudio. El muestreo se llevó a cabo en el Rancho Santa Bárbara (1874725.26 m N, $728030.54 \mathrm{~m} \mathrm{E})$, 2800 msnm, ubicado en la Sierra de Cuatro Venados, Municipio de Clavellinas, Oaxaca, México. El clima de la zona es semicálido subhúmedo, con lluvias invernales menores al 5\%, con fórmula climática $(\mathrm{A}) \mathrm{C}(\mathrm{w} 1)$ (Trejo 2004). La vegetación primaria del área fue perturbada en 1950, cuando el bosque de pino original fue sometido a 
extracción. Actualmente, la vegetación está representada por asociaciones secundarias, de las que se reconoce un bosque de encino-pino, un pastizal amacollado de $\mathrm{Mu}$ hlenbergia emersley con algunos arbustos de Bacharis conferta y un pastizal cespitoso e inundable dominado por gramíneas, ciperáceas y musgos (Sphagnum sp.). Los tipos de vegetación antes mencionados se consideraron en el trabajo como hábitats diferentes.

Cobertura vegetal. Para determinar la complejidad estructural del hábitat, en cada tipo de vegetación se establecieron aleatoriamente 15 parcelas circulares de 5.7 $\mathrm{m}$ de diámetro (Elzinga et al. 1998). En cada parcela se midió la cobertura vegetal, se contabilizó el número de estratos y la altura de cada uno de ellos, entre los cuales se consideraron: árboles, arbustos y herbáceas, también se incluyó la profundidad de la capa de hojarasca cuando estaba presente. La cobertura arbórea se midió en la parcela circular de $5.7 \mathrm{~m}$ de diámetro, mientras que para los arbustos, herbáceas y hojarasca, la parcela de muestreo fue de $1 \mathrm{~m}$ de diámetro (Elzinga et al. 1998).

Recolecta. La búsqueda de las lagartijas se llevó a cabo de forma bimestral, de abril de 2010 a marzo de 2011, en un horario de 10:00-17:00 h, de acuerdo con la actividad de los organismos de cada especie. Las lagartijas fueron recolectadas directamente con las manos (Casas-Andreu et al. 1991). De cada ejemplar capturado se registró el hábitat, el microhábitat, el sexo, y con un vernier digital (con precisión de $0.01 \mathrm{~mm}$ ) se midió la longitud total, longitud hocico cloaca (LHC) y la distancia a la que se desprendió la cola, cuando era el caso. Posterior a esto, los individuos recolectados fueron marcados (ectomización de falanges) y liberados para evitar medir la misma lagartija en los muestreos posteriores. Sólo algunos ejemplares fueron conservados como material de referencia, los que están depositados en la Colección de Anfibios y Reptiles de la UAM-Iztapalapa.

Clase de edad. Se reconocieron dos categorías de edad, jóvenes y adultos. En S. bicanthalis fueron considerados adultos los machos con una LHC $>35 \mathrm{~mm}$ (HernándezGallegos et al. 2002), en el caso de las otras dos especies no existen trabajos previos que nos indicaran la talla mínima del adulto, por lo que, algunos individuos fueron disectados para establecer la edad adulta con base en la presencia de huevos (en oviducto) o folículos vitelogénicos (en ovario) en las hembras, y con el epidídimo con producción de espermatozoides en los machos (Kumar et al. 2011).

La determinación del sexo en las especies de Sceloporus se hizo con base en el par de escamas postanales agrandadas presente en los machos y ausente en las hembras (Köhler \& Heimes 2002). En M. gadovi, la diferenciación sexual sólo fue posible en los adultos, y se usó como criterio la presencia de hemipenes, forma general de la cabeza y cuerpo, así como los patrones de color en la región ventral del cuerpo, ya que en los machos el vientre está más pigmentado de color negro que en las hembras (Good 1988).

Análisis. Las diferencias en la frecuencia de mutilación de la cola entre sexos, categorías de edad, especie y hábitat se evaluaron usando tablas de contingencia y una prueba de Chi cuadrada (Zar 1997).

\section{RESULTADOS}

Caracterización de los hábitats. El bosque de pino-encino fue el hábitat con la estructura vertical más compleja y del que se reconocieron un estrato arbóreo, un arbustivo y un herbáceo. El pastizal amacollado estuvo compuesto por un estrato arbustivo y un herbáceo, mientras que el pastizal cespitoso estuvo formado sólo por el estrato herbáceo. El estrato herbáceo tuvo una cobertura de 5.3\% en el bosque, de $62.8 \%$ en el pastizal amacollado y del $99.5 \%$ en el pastizal cespitoso. El bosque de pino-encino fue el único hábitat con una capa de hojarasca y donde los troncos caídos y tocones cubrieron el 1.6\%, mientras que en los otros dos hábitat, aunque hubo troncos y tocones, su presencia fue menor, por lo que no fue estimada (Cuadro 1).

Uso de hábitat. En los tres hábitats explorados se registraron 259 individuos de las tres especies, 101 pertenecieron a M. gadovii, 37 a S. bicanthalis y 121 a S. subpictus. La frecuencia de uso de hábitat sugiere que cada especie de lagartija prefiere un hábitat particular $\left(\chi^{2}=274.15\right.$, g.l. $=$ $4, p=0.001$; Cuadro 2). De las tres especies de lagartijas, sólo $S$. subpictus se encontró en los tres hábitats explorados, con mayor frecuencia en el pastizal amacollado. Las otras dos especies usaron dos tipos de hábitats cada una, en el caso de $S$. bicanthalis fue más frecuente en el pastizal cespitoso en comparación con el pastizal amacollado, mientras que $M$. gadovii usó más el bosque de encinopino en comparación con el pastizal amacollado (Cuadro 2). Además, cada especie utilizó con mayor frecuencia un microhábitat específico, en el caso de $S$. subpictus fue el de los troncos caídos ( $72 \%$ de los individuos), el 21\% uso el piso y el $7 \%$ restante se encontró sobre arbustos. En $S$. bicanthalis, el $82 \%$ de los individuos se encontraron sobre los pastos y el $18 \%$ restante sobre troncos caídos, 
Cuadro 1. Características físicas en tres tipos de vegetación (hábitat) analizados en Clavellinas, Oaxaca.

\begin{tabular}{|c|c|c|c|c|c|c|}
\hline \multirow[b]{2}{*}{ Estrato Vegetal } & \multicolumn{2}{|c|}{ Bosque de encino-pino } & \multicolumn{2}{|c|}{ Pastizal amacollado } & \multicolumn{2}{|c|}{ Pastizal cespitoso } \\
\hline & Altura $(\mathrm{m})$ & Cobertura (\%) & Altura (m) & Cobertura $(\%)$ & Altura $(\mathrm{m})$ & Cobertura $(\%)$ \\
\hline Arbóreo & 26.6 & 67.7 & - & - & - & - \\
\hline Herbáceo & 0.34 & 5.3 & 0.8 & 62.8 & 0.35 & 99.5 \\
\hline Troncos caídos & - & 1.6 & - & - & - & - \\
\hline
\end{tabular}

Cuadro 2. Distribución de las tres especies de lagartijas (número de individuos) por tipo de hábitat en Clavellinas, Oaxaca.

\begin{tabular}{lccc}
\hline Especie & $\begin{array}{c}\text { Bosque de } \\
\text { encino-pino }\end{array}$ & $\begin{array}{c}\text { Pastizal } \\
\text { amacollado }\end{array}$ & $\begin{array}{c}\text { Pastizal } \\
\text { cespitoso }\end{array}$ \\
\hline Mesaspis gadovii & 97 & 4 & 0 \\
Sceloporus bicanthalis & 0 & 6 & 31 \\
Sceloporus subpictus & 18 & 85 & 18 \\
\hline
\end{tabular}

mientras que $M$. gadovii se encontró en un $95 \%$ de los casos sobre la hojarasca y $5 \%$ bajo troncos.

Efecto de la cobertura vegetal. La cobertura vegetal de cada tipo de hábitat no mostró una asociación significativa con la frecuencia de mutilación de la cola en ninguna de las tres especies (M. gadovi, $\chi^{2}=0.04$, g.1. $=1, \mathrm{p}=$ $0.84 ;$ S. bicanthalis, $\chi^{2}=0.16$, g.1. $=1, p=0.68 ;$ S. subpictus, $\chi^{2}=1.95$, g.1. $=2, p=0.37$; Cuadro 3 ).

Diferencias entre clases de edad. En el caso de S. bicanthalis se consideraron machos adultos aquéllos con una LHC $\geq 35 \mathrm{~mm}$ y las hembras $\geq 37 \mathrm{~mm}$; para $S$ subpictus fueron considerados adultos los machos con una LHC $\geq$ $46 \mathrm{~mm}$ y las hembras $\geq 48 \mathrm{~mm}$; mientras que en $M$. gado$v i$, las hembras adultas midieron de LHC $\geq 70.0 \mathrm{~mm}$ y los machos $\geq 65 \mathrm{~mm}$.

Al comparar el efecto de la edad sobre la frecuencia de mutilación de la cola por especie, se encontró que sólo en $M$. gadovii, la pérdida fue significativamente mayor en adultos que en jóvenes $\left(\mathrm{X}^{2}=7.9\right.$, g.l. $\left.=1, p=0.0049\right)$. En cambio, las diferencias no fueron significativas para $S$. bicanthalis $\left(\chi^{2}=1.57\right.$, g.1. $\left.=1, p=0.21\right)$, y tampoco para S. subpictus $\left(\chi^{2}=1.49\right.$, g.1. $=1, p=0.22$; Cuadro 4$)$.
Cuadro 4. Influencia de la edad ( $\mathrm{J}$ = jóvenes; $\mathrm{A}$ = adultos) en la pérdida de la cola en tres especies de lagartijas analizadas.

\begin{tabular}{lccc}
\hline Especie & Edad & $\begin{array}{c}\text { Individuos } \\
\text { con la cola } \\
\text { mutilada }\end{array}$ & $\begin{array}{c}\text { Individuos } \\
\text { con la cola } \\
\text { completa }\end{array}$ \\
\hline Mesaspis gadovii & J & 7 & 26 \\
Sceloporus bicanthalis & J & 36 & 32 \\
Sceloporus subpictus & A & - & 11 \\
& J & 4 & 20 \\
& A & 25 & 25 \\
\hline
\end{tabular}

Diferencias entre sexos. Las diferencias en la frecuencia de mutilación entre los sexos (machos y hembras de la misma especie), sólo se evaluó en los adultos y no fueron significativas para ninguna especie (M. gadovii, $\chi^{2}=$ 2.09 , g.1. $=1, p=0.14 ;$ S. bicanthalis, $\chi^{2}=0.06$, g.1. $=1$, $p=0.80 ;$ S. subpictus, $\chi^{2}=3.52$, g.l. $\left.=1, p=0.06\right)$. No obstante, los machos de las tres especies presentaron mayor porcentaje de colas mutiladas en comparación con las hembras, y fueron respectivamente $62.85 \%$ vs. $42.42 \%$ en M. gadovii, $40.0 \%$ vs. $20.0 \%$ en S. bicanthalis y $36.95 \%$ vs. $17.39 \%$ para S. subpictus (Cuadro 5).

Diferencias entre especies. Las diferencias en la frecuencia de mutilación de la cola fue significativa entre las tres especies $\left(\chi^{2}=13.01\right.$, g.l. $\left.=2, p=0.0015\right)$. La especie con mayor porcentaje de mutilación fue $M$. gadovii con $42.6 \%$, seguida por $S$. subpictus con el $23.4 \%$ y finalmente $S$. bicanthalis con el $16.2 \%$ (Cuadro 6).

Cuadro 3. Distribución de la frecuencia de mutilación de la cola en tres especies de lagartijas por tipo de hábitat.

\begin{tabular}{lcccccc}
\hline Hábitat & \multicolumn{2}{c}{ Mesaspis gadovii } & \multicolumn{2}{c}{ Scleporus bicanthalis } & \multicolumn{2}{c}{ Sceloporus subpictus } \\
\cline { 2 - 6 } & Completa & Mutilada & Completa & Mutilada & Completa & Mutilada \\
\hline Bosque de encino-pino & 55 & 42 & - & - & 5 & 13 \\
Pastizal amacollado & 3 & 1 & 6 & 5 & 63 & 22 \\
Pastizal cespitoso & - & - & 26 & 5 & 2 \\
\hline
\end{tabular}


Cuadro 5. Influencia del sexo ( $M=$ machos; $H=$ hembras $)$ en la frecuencia de mutilación de la cola en tres especies de lagartijas adultas en Clavellinas, Oaxaca.

\begin{tabular}{lccc}
\hline Especie & Sexo & \multicolumn{2}{c}{ Cola } \\
\cline { 3 - 4 } & & completa & mutilada \\
\hline Mesaspis gadovii & M & 13 & 22 \\
& H & 19 & 14 \\
Sceloporus bicanthalis & $\mathrm{M}$ & 10 & 4 \\
& $\mathrm{H}$ & 10 & 2 \\
Sceloporus subpictus & $\mathrm{M}$ & 29 & 17 \\
& $\mathrm{H}$ & 38 & 8 \\
\hline
\end{tabular}

Cuadro 6. Distribución de la pérdida de la cola (número de individuos) en las tres especies de lagartijas analizadas.

\begin{tabular}{lcc}
\hline Especie & Cola mutilada & Cola completa \\
\hline Mesaspis gadovii & 43 & 58 \\
Sceloporus bicanthalis & 6 & 31 \\
Sceloporus subpictus & 29 & 92 \\
\hline
\end{tabular}

\section{DISCUSIÓN}

La depredación es considerada como una de las causas de mayor mortalidad en los reptiles (Martin \& López 1996; Wilson 1992). Evitar ser depredado mientras se atienden otras actividades ha favorecido la evolución de una variedad de estrategias anti-depredadoras, que en los lagartos van desde la crípsis e inmovilidad hasta la automutilación (Arnold 1988). La autotomía caudal es un mecanismo eficiente que incrementa las posibilidades de supervivencia de la presa cuando ocurre un encuentro con un depredador (Congdon et al. 1974).

Diferentes variables influyen en la probabilidad de perder la cola en una lagartija, tales como la complejidad del hábitat, el sexo, la edad, el estado reproductor, entre otros. Sin embargo, la presente investigación indica que la estructura del hábitat, el sexo o la edad del individuo (a excepción de $M$. gadovii) no influyen de manera significativa en la frecuencia de mutilación en M. gadovii, Sceloporus bicanthalis o S. subpictus. En cuanto a la estructura del hábitat, algunos autores mencionan que la cobertura vegetal y el tipo de sustrato son dos componentes importantes que eligen las presas para disminuir la depredación, debido a que la vegetación brinda sitios de refugios, lo que interfiere en la visibilidad (Biaggini et al. 2009; Eason \& Stamps 1992; Warrick et al. 1998). Sin embargo, en el presente estudio se encontró que la pérdida de la cola en las tres especies de lagartijas, no mostró diferencias significativas entre los tres hábitats, por lo tanto, ésta no es una variable importante que explique la frecuencia de mutilación en estas especies.

Los adultos de las tres especies de lagartijas estudiadas tuvieron mayor frecuencia de colas rotas en comparación con los jóvenes, no obstante las diferencias sólo fueron significativas en $M$. gadovii. Resultados similares se han reportado para Uma paraphygas (Castañeda et al. 2011) y S. scalaris (Ortega-Rubio et al. 2000). Este hecho parece reflejar la experiencia de encuentros previos de los adultos con los depredadores, además de la consecuencia de interacciones intraespecíficas que llevan a cabo los adultos cuando establecen su territorio o buscan pareja, tareas por las que los jóvenes han estado menos expuestos. Sin embargo, también cabe la posibilidad de que la menor frecuencia de colas rotas en los jóvenes sea consecuencia de ataques más efectivos por un depredador (Jacsik \& Greene 1984; Medel et al. 1988; Whiting et al. 2006).

Las diferencias entre los sexos de los adultos no fueron significativas para ninguna especie, aunque los machos de las tres especies presentaron un mayor porcentaje de colas mutiladas. Resultados similares se han observado en $S$. grammicus (Leyte-Manríquez et al. 2007) y Uma exsul (García-de la Peña et al. 2004). Este suceso puede ser consecuencia de la conducta de exhibición por parte de los machos durante la etapa de cortejo y apareamiento, así como de la conducta agonística que exhiben los machos cuando buscan acceder a las hembras o cuando establecen y defienden su territorio (Vitt et al. 1974). Durante la época reproductora, los machos exhiben sus atributos a las hembras (ejem., patrones de coloración o fortaleza física), conducta que también llama la atención de los depredadores. Asimismo, cuando contienden por las hembras son más conspicuos a los depredadores, ya que están menos atentos a su entorno, y en consecuencia, se conviertan en un blanco más fácil (Vitt 1992; Ramírez-Bautista et al. 2002; Whiting et al. 2006). También durante la competencia reproductora pueden ocurrir enfrentamientos entre machos que terminen con un animal mutilado o dañado en alguna parte de su cuerpo. Al respecto, se sabe que en Abronia vasconcelosii durante la contienda entre machos, el $78 \%$ de las mordidas van dirigidas a la cola, por lo tanto, existe mayor probabilidad de perderla si la lucha progresa (Formanowicz et al. 1990b). En un estudio con S. magister, Vitt (1974) concluye que la pérdida de la cola en los machos es más una consecuencia de la lucha intraespecífica que resultado de la depredación.

De las tres especies de lagartijas, M. gadovii fue la que tuvo un mayor porcentaje de mutilación de la cola 
en comparación con las dos especies de Sceloporus. Este hecho puede estar relacionado con la conducta agresiva que exhibe la primera especie. Como se sabe, las lagartijas de la familia Anguidae se caracterizan por su conducta agonística durante los encuentros intraespecíficos, y se ha documentado que el sitio donde más se agreden, es la cola y la cabeza (Dashevsky et al. 2013; Formanowickz et al. 1990b; García-Bastida et al. 2013). Durante la época de celo, los encuentros entre machos de $M$. gadovii aumentan, como sucede en $A$. vasconcelosii (Formanowic$\mathrm{kz}$ et al. 1990b), y en consecuencia, la probabilidad de mutilación caudal también se incrementa, lo que puede explicar la mayor frecuencia de mutilación de los adultos respecto a los jóvenes. A la mayor frecuencia de mutilación de la cola en $M$. gadovii puede agregarse el efecto de la depredación, debido a que en comparación con las especies de Sceloporus que huyeron siempre que se les intentó atrapar, y rara vez se muestran agresivas cuando se capturan, $M$. gadovii mostró un comportamiento diferente, primero intentó la huída, pero cuando su captura era inminente, siempre se defendió abriendo el hocico y tratando de morder. Suponemos que ante el acoso de un depredador, M. gadovii se comporta de manera similar, y en consecuencia existe mayor probabilidad de que pierda la cola. La frecuencia de colas mutiladas en una población de lagartijas se ha interpretado como un índice de presión de depredación (Pianka 1986; Sohoener 1979; Turner et al. 1982), argumento que Jaksic \& Greene (1984) y Medel et al. (1988) proponen que se debe interpretar como el reflejo de múltiples intentos de depredación por un depredador poco eficiente, lo que suponemos también puede estar pasando con M. gadovii.

Los resultados del estudio sugieren que el sexo, la edad del individuo (a excepción de $M$. gadovii) y la complejidad vertical del hábitat no mostraron una relación significativa con la pérdida de la cola en ninguna de las tres especies de lagartijas analizadas. Sin embargo, lo observado en el campo y en otros estudios sugiere que el comportamiento más agresivo de $M$. gadovii pudiera explicar la mayor frecuencia de autotomía caudal en esta especie en comparación con las dos especies de Sceloporus. Finalmente, es necesario un diseño experimental diferente para determinar si $M$. gadovii sufre menor depredación debido a esta conducta agresiva en comparación con las especies de Sceloporus analizadas. Así también, en estudios futuros se podría llegar a considerar la época reproductora de cada especie, debido a que dependiendo de la estación (reproductora o no reproductora) la frecuencia de mutilación de colas puede variar, ya que la intensidad del comportamiento agonístico y aun la presión de depredación podrían ser diferentes entre estaciones. Además, es necesario llevar a cabo estudios que aporten argumentos sólidos acerca de las causas que provocan la autotomía en lagartijas considerando la territorialidad y la eficiencia real de los depredadores existentes en el área de estudio.

AGRADECIMIENTOS. A las autoridades municipales de Santiago Clavellinas y a Don Wenceslao Sánchez y Familia por permitirnos el acceso al Rancho Santa Bárbara para realizar el estudio. Así también, agradecemos a dos revisores anónimos por sus sugerencias y comentarios que ayudaron a mejorar este escrito.

\section{LITERATURA CITADA}

Alibardi, L. 2010. Morphological and cellular aspects of tail and limb regeneration in lizards. Advances in Anatomy, Embryology and Cell Biology, 207: 1-109.

Arnold, E. N. 1988. Caudal autotomy as a defense. pp: 235-273. In: C. Gans \& R. B. Huey (Eds.). Biology of the Reptilia. Alan R. Liss, New York.

Ballinger, R. E., Nietfeldt, J. W. \& Krupa, J. J. 1979. An experimental analysis of the role of the tail in attaining high running speed in Cnemidophorus sexlineatus (Reptilia: Squamata: Lacertilia). Herpetologica, 35: 114-116.

Bateman, P. W. \& Fleming, P. A. 2009. To cut a long tail short: a review of lizard caudal autotomy studies carried out over the last 20 years. Journal of Zoology, 277: 1-14.

Biaggini, M., Berti, R. \& Corti, C. 2009. Different habitats, different pressures? Analysis of escape behavior and ectoparasite load in Podarcis sicula (Lacertidae) populations in different agricultural habitats. Amphibia-Reptilia, 30: 453-461.

Brown, R. M., Taylor, D. H. \& Gist, D. H. 1995. Effect of caudal autotomy on locomotor performance of wall lizards (Podarcis muralis). Journal of Herpetology, 29: 98-105.

Casa-Andreu, G., Valenzuela, G. L. \& Ramírez-Bautista, A. 1991. Cómo Hacer Una Colección de Anfibios y Reptiles. Cuaderno número 10. Instituto de Biología, Universidad Nacional Autónoma de México, 68 pp.

Castañeda, G., García-De la Peña, C., Gadsden, H., ContrerasBalderas, A. J. \& Cooper Jr., W. E. 2011. Tail loss incidence in the Chihuahuan fringe toed-lizard Uma paraphygas (Squamata: Phrynosomatidae). Revista Mexicana de Biodiversidad, 82: 193198.

Clause, A. R. \& Capaldi, E. A. 2006. Caudal autotomy and regeneration in lizards. Journal of Experimental Zoology, 305: 965-973.

Congdon, J. D., Vitt, L. J. \& King, W. W. 1974. Geckos: adaptive significance and energetics of tail autotomy. Science, 184: 13791380.

Dashevsky, D., Meik, J. M., Mociño-Deloya, E., Setser, K. \& Schaack, S. 2013. Patterns of sexual dimorphism in Mexican alligator lizards, Barisia imbricate. Ecology and Evolution, 3: 255-261.

Dial, B. E. 1984. Predator escape success in tailed versus tailless Scincella lateralis (Suaria: Scincidae). Animal Behavior, 31: 301-302. 
Dial, B. E. \& Fitzpatrick, L. C. 1981. The energetic cost of tail autotomy to reproduction in the lizard Coleonyx brevis (Sauria: Gekkonidae). Oecologia, 51: 310-317.

Eason, P. K. \& Stamps, J. A. 1992. The effect of visibility on territory size and shape. Behavioral Ecology, 3: 166-172.

Elzinga, C. L., Salzer, D. W. \& Willoughby, J. W. 1998. Measuring \& monitoring plant populations. Bureau of Land Management, U. S. Department of the Interior, $477 \mathrm{pp}$.

Formanowicz, D. R., Brodie, E. D. \& Bradley, P. J. 1990a. Behavioral compensation for tail loss in the ground skink, Scincella lateralis. Animal Behavior, 40: 782-784.

Formanowicz, D. R., Brodie, E. D. \& Campbell, J. A. 1990b. Intraspecific aggression in Abronia vasconcelosii (Sauria, Anguidae), a tropical, arboreal lizard. Biotropica, 22: 391-396.

Fox, S. F. \& McCoy, J. K. 2000. The effects of tail loss on survival, growth, reproduction, and sex ratio of offspring in the lizard Uta stansburiana in the field. Oecologia, 122: 327-334.

Fox, S. F., Conder, J. M. \& Smith, A. E. 1998. Sexual dimorphism in the ease of tail autotomy: Uta stansburiana with and without previous tail loss. Copeia, 1998: 376-382.

Fox, S. F. \& Rostker, M. A. 1982. Social cost of tail loss in Uta stansburiana. Science, 218: 692-693.

García-Bastida, M., Lazcano, D., McBrayer, L. D. \& MercadoHernández, R. 2013. Sexual dimorphism in the alligator lizard Gerrhonotus infernalis (Sauria: Anguidae): implications for sexual selection. The Southwestern Naturalist, 58: 202-208.

García-De la Peña, C., Castañeda, G., Gadsden, H., ContrerasBalderas, A. J. \& Lazcano, D. 2004. Autotomía caudal de Uma exsul (Sauria: Phrynosomatidae). Boletín de la Sociedad Herpetológica Mexicana, 12: 43-48.

Good, D.A. 1988. Phylogenetic relationships among gerrhonotine lizards: an analysis of external morphology. University of California Publications in Zoology, 121: 1-139.

Hernández-Gallegos, O., Méndez-de la Cruz, F. R., Villagrán-Santa Cruz, M. \& Andrews, R. M. 2002. Continuous spermatogenesis in the lizard Sceloporus bicanthalis (Sauria: Phrynosomatidae) from high elevation habitat of central Mexico. Herpetologica, 58: 415-421.

Jaksić, F. M. \& Greene, H. D. 1984. Empirical evidence of non-correlation between tail loss frequency and predation intensy on lizards. Oikos, 42: 407-411.

Köhler, G. \& Heimes, P. 2002. Stachelleguane. Herpeton-Verlag. Germany. 174 pp.

Kumar, S., Roy, B. \& Rai, U. 2011. Hormonal regulation on testicular functions in reptiles. Pp. 63-68. In: D. O. Norris \& K. H. López (Eds.). Hormones and reproduction of vertebrates reptiles. Academic Press, London, UK.

Leaché, A. D., Palacios, J. A., Minin, V. N. \& Bryson Jr., R. W. 2013. Phylogeography of the Trans-Volcanic bunchgrass lizard (Sceloporus bicanthalis) across the highlands of south-eastern Mexico. Biological Journal of the Linnean Society, 110: 852-865.

Leyte-Manrique, A., Ramírez-Bautista, A. \& Hernández-Salinas, U. 2007. Contribución a la ecología de Sceloporus grammicus: presencia de cola regenerada en dos poblaciones del estado de $\mathrm{Hi}-$ dalgo México. Boletín de la Sociedad Herpetológica Mexicana, 15: 13-15.

Martin, J. \& Avery, R. A. 1997. Tail loss affects prey capture deci- sions in the lizard, Psammodromus algiricus. Journal of Herpetology, 31: 292-295.

Martín, J. \& López, P. 1996. Avian predation on a large lizard (Lacerta lepida) found at low population densities in Mediterranean habitats: an analysis of bird diets. Copeia, 1996: 722-726.

Martin, J. \& Salvador, A. 1993. Tail loss reduces mating success in the Iberian rock-lizard, Lacerta monticola. Behavioral Ecology and Sociobiology, 32: 185-189.

Medel, R. G., Jiménez, J. E., Fox, S. E. \& Jacksic, F. M. 1988. Experimental evidence that high population frequencies of lizard tail autotomy indicate inefficient predation. Oikos, 53: 321-324.

Ortega-Rubio, A. \& Arriaga, L. 1990. Seasonal abundance, reproductive tactics and resource partitioning in two sympatric Sceloporus lizards (Squamata: Iguanidae) of México. Revista de Biología Tropical, 38: 491-495.

Ortega-Rubio, A., Halffter, G. \& Barbault, R. 2000. Bunch grass lizard, Sceloporus scalaris, population dynamics at La Michilia Biosphere Reserve, Mexico. Herpetological Journal, 10: 33-39.

Pianka, E. R. 1986. The ecology and natural history of desert lizards. Princeton University Press, Princeton.

Pianka, E. R. \& Vitt, L. J. 2003. Lizards: windows to the evolution of diversity. University of California Press, California, USA. 333 pp.

Punzo, C. M. 1982. Tail autotomy and running speed in the lizards Cophosaurus texanus and Uma notata. Journal of Herpetology, 16: 331-332.

Ramírez-Bautista, A., Ramos-Flores, O. \& Sites Jr., J.W. 2002. Reproductive cycle of the spiny lizard Sceloporus jarrovii (Sauria: Phrynosomatidae) from north-central México. Journal of Herpetology, 36: 225-233.

Salvador, A., Martin, J., Lopez, P. \& Veiga, J. P. 1996. Long-term effect of tail loss on home range size and access to females in male lizards, Psammodromus algiricus. Copeia, 1996: 208-209.

Schoener, T. W. 1979. Inferring the properties of predation and other injury-producing agents from injury frequencies. Ecology, 60: $1110-1115$

Trejo, I. 2004. Clima. pp. 67-85. In: A. J. García-Mendoza, M. J. Ordóñez \& M. A. Briones-Salas (Eds.). Biodiversidad de Oaxaca. Instituto de Biología, UNAM. Fondo Oaxaqueño Para la Conservación de la Naturaleza-World Wildlife Fund, México.

Turner, F. B., Medica, P. A., Jennrich, R. I. \& Maza, B. G. 1982. Frequency of broken tails among Uta stansburiana in southern Nevada and a test of the predation hypothesis. Copeia, 1982: 835840.

Vitt, L. J. 1981. Tail autotomy and regeneration in the tropical skink, Mabuya heathi. Journal of Herpetology, 15: 454-457.

Vitt, L. J. 1992. Diversity of reproduction strategies among Brazilian lizards and snakes: the significance of lineage and adaptation. Pp. 135-149. In: W.C. Hamlett (Eds.). Reproductive biology of South American vertebrates. Springer-Verlag, New York.

Vitt, L. J. \& Caldwell J. P. 2009. Herpetology an Introductory Biology of Amphibians and Reptiles. $3^{\circ}$ edición. Elsevier Inc. 697 pp.

Vitt, L. J., Congdon, J., Hulse, A. C. \& Platz, J. E. 1974. Territorial aggressive encounters and tail breaks in the lizard Sceloporus magister. Copeia, 1974: 990-993.

Warrick, G. D., Kato, T. T. \& Rose, B. R. 1998. Microhabitat use and home range characteristics of Blunt-nosed leopard lizards. Journal of Herpetology, 32: 183-191. 
Whiting, M. J., Stuart-Fox, D. M., O'connor, D., Firth, D., Bennet, N. C. \& Blomberg, S. P. 2006. Ultraviolet signals ultra-aggression in a lizard. Animal Behaviour, 72: 353-363.

Wiens, J. J., Kuczynski, C. A., Arif, S. \& Reeder, T. W. 2010. Phylogenetic relationships of phrynosomatid lizards based on nu- clear and mitochondrial data, and a revised phylogeny for Sceloporus. Molecular phylogenetics and evolution, 54: 150-161.

Wilson, B. S. 1992. Tail injuries increase the risk of mortality in freeliving lizards (Uta stansburiana). Oecologia, 92: 145-152.

Zar, J. 1997. Biostatiscal analysis. Prentice Hall, Englewood Cliffs. 